\title{
Avaliação da prevalência de terceiros molares inferiores inclusos e da posição e inclinação do seu longo eixo em radiografias panorâmicas
}

\author{
Fernanda Sampaio N ery ${ }^{1}$ \\ Luzivalter Dias Santos ${ }^{2}$ \\ Viviane Almeida Sarmento ${ }^{3}$ \\ Edmar J osé B orges Santana ${ }^{4}$
}

\begin{abstract}
Resumo
Asinclusões dentárias mais freqüentes ocorrem em dentes quefazem erupção em épocamaistardia, ou seja, osterceiros molares, sen do a falta de espaço a principal causa deuma inclusão. A classificação dosterceiros molares inclusoséum importanteauxílio na comunicação entre profissionais, na determinação do tratamento eno planejamento cirúrgico. A amostra desteestudo foi composta por 250 radiografias panorâmicas, nas quaisum total de 70 terceiros molaresinclusos foi avaliado. A análise foi feita em uma clinica odontológica na cidade deSão Raimundo N onato - PI, no período de julho anovembro de 2004. A maioria dos dentes inclusos estava em posição mesioangulada, segundo a classificação de W inter (quarenta casos - 57,14\%). D eacordo com a classificação de Pell \& G regory, os tercei ros molares inclusos pertenciam, na sua maioria, àsclassesB ell: quarenta ecinco casos, 64,28\%, ecinqüenta eseiscasos, $70 \%$, respectivamente.
\end{abstract}

Palavras-chave: terceiro molar - classificação; impactação dentária; radiografia panorâmica.

\section{INTRODUÇÃO}

D entes retidos são aqueles que, chegada a época da erupção, não irrompem, podendo manter ou não comunicação com a cavidade bucal (MARZOLA, 1988). A retenção pode ter causas mecânicas, como a presença de um dente (DEBONI; GREGORI, 1990; BASILE; GREG O RI, 2004; H ATTAB; ALH AIJA, 1999; HATTAB; RAWASH DEH; FAH MY, 1995), condições do tecido ósseo de revestimento, espessura e densidade dos tecidos moles sobrejacentes (O LASO JI; O D U SAN YA, 2000), ou razões patológicas. A etiologia da impacção está relacionada principalmente à falta de espaço disponível na região (GANSS et al., 1993; OLIVE; BASFORD, 1981; RICH ARD SON, 1977), característica acentuada na população moderna, que parece apresentar menor crescimento ósseo, decorrente das alterações dos hábitos alimentares e melhores condições de saúde bucal (TRAIN A, 2004). Fatores sistêmicos também podem estar associados à etiologia das impacções, como Disostose cleidocraniana, Síndrome de Down, raquitismo e distúrbios endócrinos (GREG ORI, 2004).

\footnotetext{
${ }^{1}$ M estranda. Programa de Pós-graduação em O dontologia. Faculdade de O dontologia - UFBA. Salvador - BA

${ }^{2}$ Especialista em Radiologia $O$ dontológica. Centro Baiano de Estudos $O$ dontológicos. Salvador - BA

${ }^{3}$ Professora Adjunto D outora em Estomatologia Clínica. Faculdade de O dontologia - UFBA. Salvador - BA

${ }_{4}^{4}$ Professor Adjunto Doutor em Patologia Bucal. Faculdade de O dontologia - UFBA. Salvador - BA
}

\section{Correspondência para / Correspondence to:}

Fernanda Sampaio N ery

Faculdade de 0 dontologia - UFBA

Rua Arájo Pinho 62 - Canela

40.110.150 Salvador - BA - Brasil

Tel.: (71) 3336-5776

E-mail: nandasnery@hotmail.com 
O s terceiros molares são, entre todos os dentes, os que apresentam maior freqüência de impactação (TRAIN A, 2004; VASCO N CELLO S et al., 2003; SEN GUPTA et al., 1999; H AT TAB; RAWASH DEH ; FAH M Y, 1995; NEVILLE et al., 1995; MELO; ARAÚJO, 1996; PETERSON et al., 1996; GRAZIAN I, 1995), atingindo valores em torno de $90 \%$ (BASILE; GREG ORI, 2004). A avaliação radiográfica dos terceiros molares tem fundamental importância para o estabelecimento de terapêuticas de acompanhamento e planejamento cirúrgico. Muitos dos fatores que influenciam na decisão de extração desses dentes são revelados pela avaliação radiográfica pré-operatória (W H AITES, 2003). Assim sendo, os achados radiográficos são relevantes no prognóstico de dificuldades na extração de dentes retidos (WH AITES, 2003) e, nesse sentido, a radiografia panorâmica é extremamente útil.

A literatura é rica em classificações de dentes inclusos, o que uniformiza e facilita a comunicação científica e profissional. As classificações possibilitam a transferência de informações e de experiências, gerando metodização dos estudos, e devem ser priorizadas para o estabelecimento da técnica cirúrgica mais adequada (TRAIN A, 2004).

As classificações mais utilizadas para dentes inclusos são a de W inter e a de Pell \& Gregory. A classificação de W inter é uma normativa de décadas, que, não obstante inúmeras mudanças terem ocorrido ao longo do tempo, é considerada a ideal por Ferreira e Serra (1990). Essa classificação avalia o longo eixo do terceiro molar em relação ao longo eixo fisiológico do segundo molar inferior. D essa forma, são encontradas as seguintes posições (W INTER, 1926; FARIAS et al., 2003):

- Vertical - quando os eixos estiverem paralelos.

- M esioangular - quando o longo eixo do terceiro molar estiver em posição medial em relação ao longo eixo do segundo molar.

- D istoangular - quando o longo eixo do terceiro molar estiver em posição distal em relação ao longo eixo do segundo molar.

- H orizontal - quando o dente que está totalmente deitado, com a face oclusal voltada para o segundo molar, estando suas raízes para a distal.

A classificação de Pell $\&$ Gregory determina a posição do dente incluso, considerando a profundidade no osso e o espaço existente entre a distal do segundo molar e o ramo mandibular. É uma classificação interessante, do ponto de vista da avaliação cirúrgica, no que concerne à remoção de osso no sentido anterior e posterior (FERREIRA eSERRA, 1990). Pell e Gregory (1933) classificaram os dentes retidos em relação ao plano oclusal do segundo molar, em classes $A, B$ e C; e em relação à borda anterior do ramo mandibular, para os terceiros molares inferiores, em classes 1, 2 e 3 (FARIAS et al., 2003), como é detalhado a seguir:

- Classe 1 - se houver espaço entre a distal do segundo molar e a borda anterior do ramo da mandíbula, ou seja, quando o diâmetro mesio-distal da coroa do terceiro molar está completamente à frente do bordo.

- Classe 2 - se houver espaço entre a distal do segundo molar e a borda anterior do ramo da mandíbula, mas este é insuficiente.

- Classe 3 - na inexistência de espaço entre a distal do segundo molar e a borda anterior do ramo da mandíbula, ou quando o diâmetro mesio-distal da coroa do terceiro molar está completamente dentro do ramo.

- Classe A - quando a face oclusal do terceiro molar está no mesmo plano ou acima do segundo molar.

- Classe B - quando a face oclusal do terceiro molar está entre o nível oclusal e cervical;

- Classe C - face oclusal do terceiro molar está abaixo da linha cervical do segundo molar.

0 presente estudo tem como objetivo estimar a prevalência de terceiros molares inferiores inclusos, categorizando-os em relação à classificação de W inter e de Pell \& G regory, em radiografias panorâmicas realizadas em uma clínica de radiologia odontológica da cidade de São Raimundo N onato - Piauí.

\section{MATERIAIS E MÉTODOS}

\section{População e amostra}

Após aprovação do projeto desta pesquisa pelo Comitê de Ética em Pesquisa da Facul- 
dade de $O$ dontologia da Universidade Federal da Bahia, foram selecionadas as primeiras duzentas e cinqüenta radiografias panorâmicas, que apresentaram qualidade suficiente para 0 diagnóstico. Essas radiografias foram realizadas em uma Clínica de Radiologia O dontológica privada, da cidade de São Raimundo N onato PI. Após análise, as radiografias foram entregues aos pacientes, evitando-se uma nova exposição. O s pacientes cujas radiografias foram selecionadas para o estudo assinaram o Termo de Consentimento Esclarecido elaborado para esta pesquisa, permitindo a utilização do referido exame.

\section{Coleta e instrumento de coleta de dados}

A radiografia panorâmica foi realizada de forma padronizada, utilizando-se o aparelho Rotograph Plus (70 kV p e 10 mA; Villa Sistemi M edicali, M ilano - Italy) e filmes radiográficos (T-M at G/R - Kodak; Eastman Kodak Company, N ew York, USA), associados a placas intensificadoras (Lanex regular - Kodak). Os pacientes foram protegidos com colete de chumbo durante a tomada radiográfica. A pós a exposição, os filmes foram colocados em uma processadora automática (AT 2000, Air T hechnics, USA) e posteriormente identificados. Classificação do terceiro molar quanto a relação entre os planos oclusais do 30 e 20 molar de acordo com Pell e Gregory.

A análise radiográfica realizou-se em ambiente escurecido e silencioso, sobre um negatoscópio de luz fixa (Firefly $n^{\circ} 4, \mathrm{H}$ itco, $\mathrm{H}$ iltrade $\mathrm{C}$ o., Ltd., H ong Kong), com auxílio de lupa de aumento de duas vezes e máscaras de papel preto, por um radiologista experiente. Foram utilizadas fichas específicas para as anotações do resultado da análise. Foi pesquisada a presença de terceiros molares inferiores inclusos, e o(s) lado(s) comprometido(s) fora(m) registrado(s), assim como a idade e gênero de cada indivíduo. O s terceiros molares inferiores inclusos ainda foram submetidos à classificação de W inter e de Pell \& G regory.

Para a análise estatística, foram calculadas as freqüências absolutas e relativas das variáveis estudadas.

\section{RESULTADOS}

Foram avaliadas duzentos e cinqüenta radiografias panorâmicas. D essas, noventa radiografias foram excluídas, por pertencerem a indivíduos com menos de 18 anos de idade, pois nesses indivíduos terceiros molares em processo de erupção poderiam ser confundidos com dentes retidos. Ao final, cento e sessenta radiografias panorâmicas participaram do estudo.

Em relação ao gênero, cento e nove radiografias $(68,13 \%)$ pertenciam a indivíduos do gênero feminino, enquanto apenas cinqüenta e uma $(31,87 \%)$ a indivíduos do gênero masculino, conforme mostrado na Figura 1.

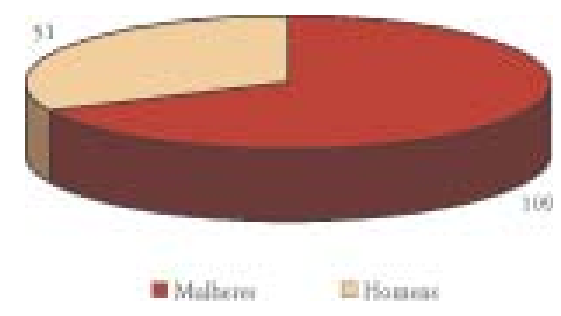

Figura 1- D istribuição da amostra em relação ao gênero

A faixa etária mais prevalente foi a de 18 a 27 anos $(65,62 \%)$, seguida daquela de 28 a 37 anos $(26,25 \%)$ e 38 a 47 anos (6,25\%). Os dados são exibidos na Figura 2.

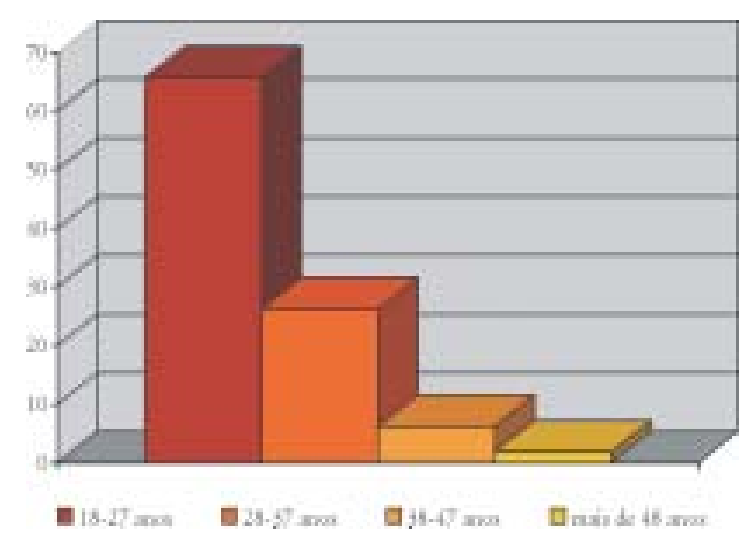

Figura 2- D istribuição da amostra em relação à faixa etária 
$D$ as cento e sessenta radiografias que fizeram parte do estudo, quarenta e seis $(28,75 \%)$ apresentavam terceiros molares inferiores inclusos (FIGURA 3). Dessas, trinta e quatro $(73,91 \%)$ pertenciam a indivíduos do gênero feminino e doze $(26,09 \%)$ ao gênero masculino. O s resultados são mostrados na Figura 4.

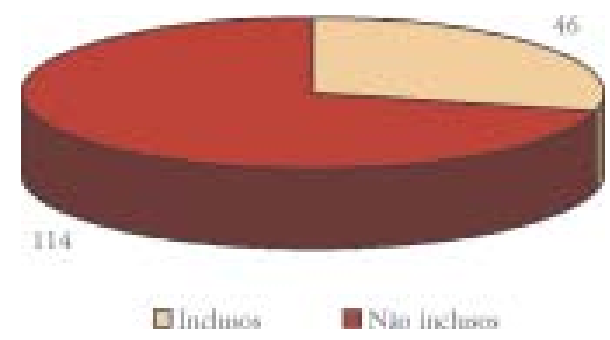

Figura3 - D istribuição da amostra segundo a presença ou não deterceiros molaresinferioresinclusos
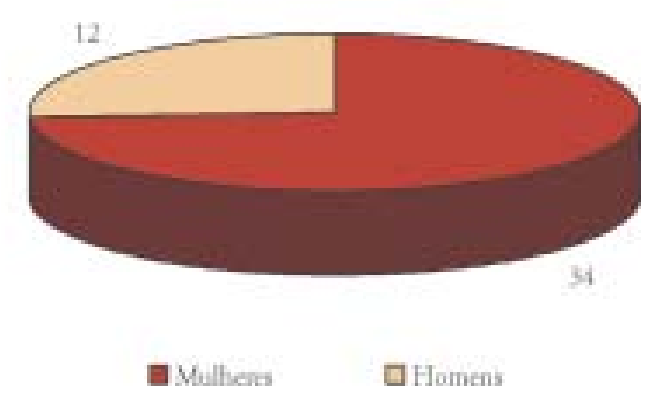

Figura4 - D istribuição dasradiografias com terceirosmolaresinclusos, em relação ao gê nero

$\mathrm{N}$ as quarenta e seis radiografias com terceiros molares inferiores inclusos, existiam setenta inclusões, sendo trinta e nove $(55,71 \%)$ de terceiros molares inferiores esquerdos (dente 3.8) e trinta e uma $(44,29 \%)$ de terceiros molares inferiores direitos (dente 4.8). Esses dados podem ser vistos na Figura 5.

Categorizados em relação à classificação de W inter, observou-se que a maioria dos terceiros molares inclusos estava em posição mesioangulada (quarenta casos - 57,14\%), seguida da posição vertical (quinze casos $21,43 \%$ ) e das posições distoangulada (oito
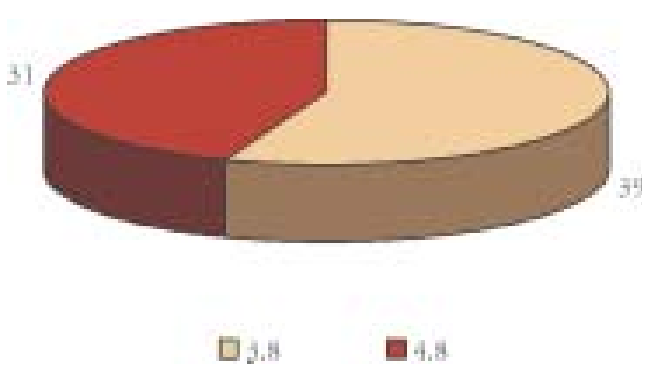

Figura 5 - N úmero deterceirosmolaresinferiores inclusos, quanto à hemiarcada.

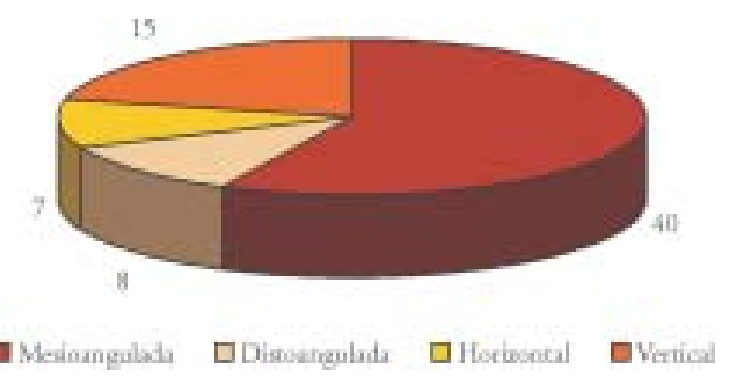

Figura 6 - D istribuição dosterceiros molares inclusos, em relação à C lassificação deW inter

casos - 11,42\%) e horizontal (sete casos - 10\%) (FIGURA 6).

Analisando separadamente os dentes inclusos, observou-se que a maioria dos terceiros molares inferiores esquerdos (3.8) e direitos (4.8) estava em posição mesioangulada, sendo vinte e cinco dentes do lado esquerdo e quinze do lado direito. $\mathrm{N}$ a posição vertical, foram encontrados sete casos do lado esquerdo e oito do lado direito; na posição distoangulada, três dentes do lado esquerdo e cinco do lado direito; e na posição horizontal, quatro do lado esquerdo e três do lado direito. Os dados são mostrados na Tabela 1.

Já em relação à classificação de Pell \& Gregory, os terceiros molares inclusos pertenciam às classes B e II, na sua maioria (quarenta e cinco casos -64,28\%; e cinqüenta e seis casos $70 \%$, respectivamente), seguidos das classes $A$ (dezenove casos - 27,14\%), I (quatorze caso $20 \%$ ), C (seis casos - 8,57\%), nessa ordem, como mostrado nas Figuras 7 e 8. 
Tabela 1 - Distribuição dos terceiros molares inclusos, em relação à Classificação de Winter

\begin{tabular}{l|c|c|c}
\hline \multicolumn{1}{c|}{ Posição } & Dente 3.8 & Dente 4.8 & Total \\
\hline Mesioangulado & $\begin{array}{c}25 \\
(64,10 \%)\end{array}$ & $\begin{array}{c}15 \\
(48,39 \%)\end{array}$ & 40 \\
\hline Vertical & $\begin{array}{c}7 \\
(25,64 \%)\end{array}$ & $\begin{array}{c}8 \\
(25,80 \%)\end{array}$ & 15 \\
\hline Distoangulado & $\begin{array}{c}3 \\
(7,69 \%)\end{array}$ & $\begin{array}{c}5 \\
(16,13 \%)\end{array}$ & 8 \\
\hline Horizontal & $\begin{array}{c}4 \\
(10,26 \%)\end{array}$ & $\begin{array}{c}3 \\
(9,68 \%)\end{array}$ & 7 \\
\hline Total & $\begin{array}{c}39 \\
(100 \%)\end{array}$ & $\begin{array}{c}31 \\
(100 \%)\end{array}$ & 70 \\
\hline
\end{tabular}

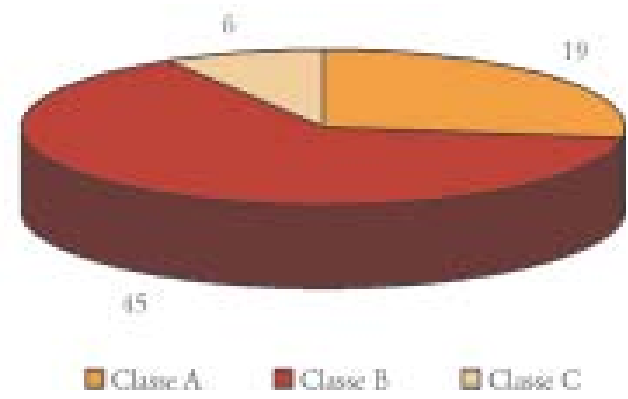

Figura 7- D istribuição dos terceiros molares inferiores inclusos, em relação à classificação de Pell \& Gregory

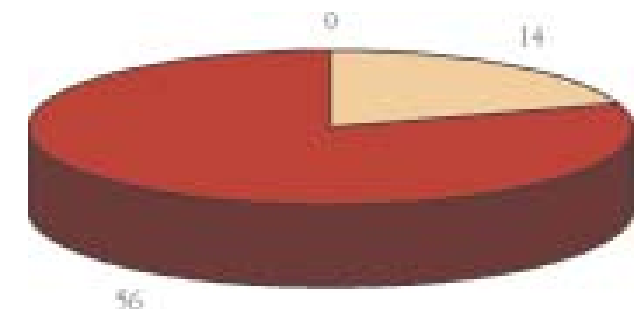

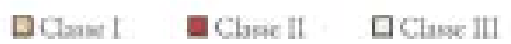

Figura 8- D istribuição dos terceiros molaresinferiores inclusos, emrelação à classificação de Pell \& Gregory

A nalisado separadamente, observou-se que 0 dente 3.8 pertencia principalmente à classe $B$ (vinte e quatro casos - 61,54\%) e à classe II (trinta casos - 76,92\%). D e forma semelhante, o dente 4.8 pertencia em sua maioria à classe $B$ (vinte e um casos - 67,74\%) e à classe II (vinte e seis casos 83,88\%). O s dados são exibidos na Tabela 2 .

Tabela 2 - Distribuição dos terceiros molares inclusos, em relação à Classificação de Pell \& Gregory, classe A, B e C.

\begin{tabular}{c|c|c|c}
\hline Classe & $\mathrm{A}$ & $\mathrm{B}$ & $\mathrm{C}$ \\
\hline Dente 3.8 & $\begin{array}{c}10 \\
(25,64 \%)\end{array}$ & $\begin{array}{c}24 \\
(61,54 \%)\end{array}$ & $\begin{array}{c}5 \\
(12,82 \%)\end{array}$ \\
\hline \multirow{2}{*}{ Dente 4.8} & 9 & 21 & 1 \\
& $(29,03 \%)$ & $(67,74 \%)$ & $(3,23 \%)$ \\
\hline Total & 19 & 45 & 6 \\
& $(27,14 \%)$ & $(64,29 \%)$ & $(8,57 \%)$ \\
\hline
\end{tabular}

Tabela 3 - Distribuição dos terceiros molares inclusos, em relação à Classificação de Pell \& Gregory, classe I, II e III

\begin{tabular}{c|c|c|c}
\hline Classe & I & II & III \\
\hline Dente 3.8 & $\begin{array}{c}9 \\
(23,08 \%)\end{array}$ & $\begin{array}{c}30 \\
(76,92 \%)\end{array}$ & - \\
\hline Dente 4.8 & $\begin{array}{c}5 \\
(16,13 \%)\end{array}$ & $\begin{array}{c}26 \\
(83,87 \%)\end{array}$ & - \\
\hline Total & $\begin{array}{c}14 \\
(20 \%)\end{array}$ & $\begin{array}{c}56 \\
(80 \%)\end{array}$ & - \\
\hline
\end{tabular}

\section{DISC USSÃO}

Em relação ao total da amostra, 68,13\% pertenciam ao gênero feminino e 31,87\% pertenciam ao gênero masculino. Esses dados refletem os resultados da maioria dos estudos de prevalência em humanos, na área odontológica, nos quais a maioria dos indivíduos pertence ao gênero feminino. I sso sugere uma preocupação maior das mulheres com sua saúde e sua aparência.

D as radiografias analisadas, 46 apresentaram terceiros molares inferiores inclusos, sendo $73,91 \%$ pertencentes a pacientes do gênero feminino, e $26,09 \%$ pertencentes ao gênero masculino. O s resultados estão de acordo com outros estudos que mostram maior prevalência de dentes inclusos no gênero feminino (VASCO N CELLO S et al., 2002; FARIAS et al., 2003; TRAIN A, 2004). Vale ressaltar que, nesses estudos, a diferença percentual entre os gê neros é pequena, quando comparada à grande 
variação do presente estudo. Esse fato pode ser explicado pelo pequeno tamanho da amostra utilizada (160 radiografias), quando comparada com as amostras dos estudos citados, cujos tamanhos variaram de 450 a 820 radiografias panorâmicas. Em contrapartida, Vasconcellos e colaboradores (2003), avaliando a ocorrência de dentes impactados em 530 radiografias panorâmicas, observaram que houve predominância no gênero masculino, com uma diferença de $3,74 \%$, em relação ao feminino.

É de comum acordo, na literatura atual, que os terceiros molares são os dentes que mais comumente sofrem impacção óssea, em adultos e adolescentes. 0 s terceiros molares inferiores, objeto deste estudo, são apontados como os dentes que apresentam maior freqüência de retenção, seguidos dos terceiros molares supe riores (WEISM AN N ; ROSA, 1990; GRAZIANI, 1995; GREGORI, 2004).

No presente estudo, $28,75 \%$ (46) do total de radiografias da amostra apresentou terceiros molares inferiores inclusos, com um total de 70 inclusões, pois, em alguns casos, houve mais de um dente incluso por radiografia. D os terceiros molares inferiores inclusos, $55,71 \%$ eram da hemiarcada esquerda (dente 3.8) e $44,29 \%$ da direita (dente 4.8). N 0 estudo de Vasconcellos e colaboradores (2003), o percentual de dentes inclusos foi maior $(52,83 \%$ - 280 radiografias). Entretanto, cabe ressaltar que, neste estudo, foram avaliados todos os tipos de impacções, incluindo os dentes supranumerários. Ainda com relação a este estudo, os terceiros molares inferiores foram os dentes mais freqüentemente impactados, representando um total de $59,57 \%$ dos dentes impactados avaliados.

A avaliação do posicionamento de terceiros molares é de extrema importância para 0 devido planejamento cirúrgico do caso, avaliação do grau de dificuldade do procedimento cirúrgico, correto diagnóstico e seleção do plano de tratamento. Alguns estudos têm sido feitos no sentido de avaliar a prevalência do posicionamento dos terceiros molares inclusos a partir das classificações de W inter e a de Pell \& Gregory (GARCIA-GODOY, 1980;
VASCON CELLOS et al., 2002; FARIAS et al., 2003). A literatura reporta também estudos que utilizam tais classificações para 0 acompanhamento de mudanças de posição de terceiros molares inferiores, ao longo do tempo (HATTAB; JORDAN, 1997).

No presente estudo, foram utilizadas as classificações de Winter e a de Pell \& Gregory para avaliação do posicionamento de terceiros molares inferiores. O bservou-se que, usando a classificação de W inter, $57,14 \%$ dos casos estavam em posição mesioangulada, seguidos da posição vertical $(21,43 \%)$. Vasconcellos e colaboradores (2002), em estudo com 450 radiografias panorâmicas, avaliaram a prevalência de terceiros molares retidos em relação à classificação de W inter. 0 s resultados demonstraram que as posições mais freqüentes foram a mesioangulada e a vertical. I mportante relatar que esses autores avaliaram o posicionamento de terceiros molares superiores e inferiores, sendo a posição vertical dos terceiros molares superiores, em ambos os gêneros, a mais prevalente. Q uando avaliados os terceiros molares inferiores, nos diferentes gêneros, observou-se que a posição mais prevalente, no gênero masculino, foi a vertical, o que diverge do presente estudo. $\mathrm{N} o$ gênero feminino, a posição mais prevalente dos terceiros molares inferiores foi a mesioangulada, dados em concordância com o presente estudo. Farias e colaboradores (2003), avaliando a prevalência de dentes inclusos em 88 prontuários na Universidade Estadual de Feira de Santana, encontraram que as posições vertical e mesioangulada foram as mais prevalentes, com uma freqüência de $36,9 \%$ e $32 \%$, respectivamente.

No presente estudo, em relação à classificação de Pell \& G regory, a maioria dos terceiros molares inclusos pertenceram às classes $B$ $(64,28 \%)$ e II (70\%). 0 estudo de Farias e colaboradores (2003) apresentou maior percentual de terceiros molares inferiores inclusos pertencentes à classe II $(72,8 \%)$, e as classes A e B apresentaram valores próximos entre si $(47,6 \%$ e 43,7\%, respectivamente).

Cabe aqui relatar o estudo de $\mathrm{H}$ attab e Jordan (1997), no qual, através de exames 
radiográficos padronizados iniciais e após quatro anos, avaliou-se a mudança da posição de terceiros molares inferiores em estudantes universitários, com média de idade 19,7 anos. Os resultados mostraram que, no momento inicial, $22 \%$ dos dentes era classe B e $78 \%$, classe $C$, o que difere do presente estudo. Após quatro anos, os novos resultados apresentaram maior concordância com este estudo, sendo que $42 \%$ dos terceiros molares impactados foram classificados como classe A, 20\%, classe B e 37\%, classe C. O s autores observaram que, dos 46 dentes classificados inicialmente como classe $C$, $37 \%$ passaram a classe A e $15 \%$ a classe B. Esses resultados permitem inferir que os dentes sofrem modificações no seu posicionamento com o tempo e que a análise radiográfica deve ser realizada em período concomitante à avaliação realizada, independentemente da sua finalidade clínica.

\section{CONCLUSÕES}

- N a amostra avaliada, a prevalência de terceiros molares inferiores inclusos foi de $28,75 \%$ (quarenta e seis casos).

- A maioria dos dentes inclusos pertencia a indivíduos do gênero feminino (73,91\% trinta e quatro casos).

- Dentre os terceiros molares inferiores inclusos, trinta e nove $(55,71 \%)$ pertenciam à hemiarcada esquerda (dente 3.8) e trinta e um $(44,29 \%)$ à hemiarcada direita (dente 4.8$)$.

- A maioria dos dentes inclusos estava em posição mesioangulada, segundo a classificação de W inter (quarenta casos - 57,14\%).

- D e acordo com a classificação de Pell \& Gregory, os terceiros molares inclusos pertenciam, na sua maioria, às classes B e II (quarenta e cinco casos - $64,28 \%$; e cinqüenta e seis casos $70 \%$, respectivamente).

\title{
Evaluation of prevalence of non erupted lower third molar and of the position and inclination of itslong axisin panoramic radiographs
}

\begin{abstract}
The enclosed teeth are more comum with teeth that don't erupt in correct time. The third molars are the most frequent impacted teeth and is probably caused by the lack of space. The classifications for impacted third molars are important for comunication with dentists, to make decicions about the cirurgic treatment, tchiniques of remotios and treatment plan. In this study, 250 panoramic radiographs are assessad. The results founded 70 impacted mandibular third molars. The analise was made in a odontologic clinic in São Raimundo N onato-PI city, at the period of july at november, 2004. The most of enclosed teeth were on mesial angulation by Winter's classification (fourty cases- $57,14 \%$ ), and by Pell \& G regory's classification the most of third mandibular molars were classes B and II ( fourty five cases - 64,28\%; and fifty six cases - $70 \%$, respectively).
\end{abstract}

Keywords. Third molars-classification, enclosed teeth, panoramic radiographs.

\section{REFERÊNCIAS}

BASILE, J.N.; GREGORI, C. Dentes inclusos. In: GREGORI, C.; CAM POS, A.C. Cirurgia buco-dento-alveolar. 2 ed. São Paulo: Sarvier, 2004.
DEBONI, M.C.Z.; GREGORI, C. Aferição das posições prevalentes dos terceiros molares inclusos. R. 0 dontol. Univ. São Paulo, São Paulo, v.4, n.2, p.87-91, 1990. 
FARIAS, J.G. et al. Prevalência de dentes inclusos em pacientes atendidos na disciplina de Cirurgia do Curso de 0 dontologia da Universidade Estadual de Feira de Santana. Pesq. Bras. 0 dontopediatr. Clín. Integr., João Pessoa, v.3, n.2, p.15-19, jul./dez. 2003.

FERREIRA, C.L.D.; SERRA, M.A. Roteiro para cirurgia dos dentes impactados: princípios gerais das técnicas e táticas cirúrgicas. 1990. Disponível em: «www.medcenter.com> Acesso em: jan. 2006.

GAN SS, C. et al. Prognosis of third molar eruption. O ral Surg, 0 ral M ed. 0 ral Pathol. 0 ral Radiol. Endod., St. Louis, v.76, n.6, p.688-693, 1993.

GARCIA-GODOY, F. A radiographic technique for maxillary third molars. J. Am. D ent. Assoc., Chicago, v.100, n.4, p.565-566, 1980.

GRAZIANI, M . Cirurgia buco-maxilo-facial. 7.ed. Rio de Janeiro: G uanabara Koogan, 1995.

GREGORI, C. Cirurgia buco-dento-alveolar. São Paulo: Sarvier, 2004.

HATTAB, F.N.; ALHAIJA, E.S.J.A. Radiographic evaluation of mandibular third molar eruption space. 0 ral Surg. 0 ral M ed. 0 ral Pathol. O ral Radiol. Endod., St. Louis, v.88, n.3, p.285-291, 1999.

HAT TAB, F.N .; JORDAN, I. Positional changes and eruption of impacted mandibular third molars in young adults. O ral Surg. 0 ral Med. O ral Pathol., St. Louis, v.84, n.6, p.604608, 1997.

HATTAB, F.N.; RAWASHDEH, M.A.; FAH M Y, M S. I mpaction status of third molars in Jordanian students. O ral Surg. O ral Med. $O$ ral Pathol. O ral Radiol. Endod., St. Louis, v.79, n.1, p.24-29, 1995.

MARZO LA, C. Retenção dental. São Paulo: Pancast, 1988.

MELO, R.E.V.A.; ARAUJO , F.R.G. Avaliação das inclusões dentárias em 92 pacientes da disciplina de cirurgia bucofacial do curso de 0 dontologia da UFPE. R. O donto Ciênc., Porto Alegre, v.11, n.22, p.7-19, 1996.
NEVILLE, B.W. et al. Patologia oral $\boldsymbol{\alpha}$ maxilofacial. Rio de Janeiro: Guanabara Koogan, 1995.

OLASOJI, H.O.; ODUSANYA, S.A. Comparative study of third molars impaction in rural and urban areas of South-W estern Nigeria. 0 dontostomatol. Trop., D akar, v.23, n.90, p.25-28, 2000.

OLIVE, R.J .; BASFORD, K.E. Transverse dento-skeletal relationships and third molar impaction. Angle 0 rthod., Appleton, v.51, p.41-47, 1981.

PELL, G.J.; GREGORY, G.T. Impacted third molars: classification and modified technique for removal. D ent. Dig., Pittsburgh, v.39, p.330-338, 1933.

PETERSON, L.J. et al. Cirurgia oral e maxilofacial contemporânea. 2.ed. Rio de Janeiro: Guanabara Koogan, 1996.

RICHARDSON, M.E. The etiology prediction of mandibular third molar impaction. Angle $\mathbf{0}$ rthod., Appleton, v.47, p.165-172, 1977.

SEN GUPTA, A. et al. The effects of dental wear on third molar eruption and on the curve of Spee in human archaeological dentitions. Arch. 0 ral Biol., 0 xford, v.44, n.11, p.925934, 1999.

TRAIN A,A.A. Estudo radiográfico das características dos terceiros molares e suas correlações com a impacção óssea. 2004. 174f. D issertação (M estrado)-Faculdade de 0 dontologia, Universidade de São Paulo, São Paulo, 2004. VASCONCELLOS, R.J.H. et al. Incidência dos terceiros molares retidos em relação à classificação de Winter. R. C ir. Traumatol. BucoM axilo-Fac., Recife, v.2, n.1, p.43-47, jan./jun. 2002.

VASCO N CELLOS, R.J.H. et al. O corrência de dentes impactados. R. Cir. Traumatol. Buco-M axilo-Fac., Recife, v.3, n.1, p.43-47, jan./mar. 2003.

WEISM AN N, R.; ROSA, E.L.S. Transplante autógeno de germe dental: considerações e re lato de caso. R. $\mathbf{O}$ donto $\mathbf{C i e ̂ n c . , ~ P o r t o ~ A l e g r e , ~}$ v.2, n.3, p.7-11, jun.1990. 
WHAITES, E. Princípios de radiologia odontológica. 3.ed. Porto Alegre: Artmed, 2003.

WINTER, G.B. Principles of exodontias as applied to the impacted third molar: a com- plete treatise on the operative technique with clinical diagnosis and radiographic interpretations. St Louis: American M edical Books, 1926. 